\title{
Calculation of aortic regurgitation orifice area by Doppler echocardiography: an application of the continuity equation
}

\author{
Alan C Yeung, Ted Plappert, Martin G St John Sutton
}

\begin{abstract}
The evaluation of aortic regurgitation by current echocardiographic techniques has been qualitative and load-dependent. The area of the regurgitant orifice, which is theoretically independent of haemodynamic conditions, has not been determined non-invasively. In 20 patients with various degrees of aortic regurgitation, this area was determined by use of the continuity equation applied during diastole. The velocity-time integrals were determined at the supravalvar (VTI $)$ and regurgitant orifice (VTI $)$ levels by pulsed and continuous wave Doppler respectively. The cross sectional area at the supravalvar level $\left(A_{s}\right)$ was also measured by cross sectional echocardiography. The regurgitant orifice is given by: $\left(A_{s} \times V I_{s}\right) / V T I_{i}$. Other noninvasive measurements of the aortic regurgitation severity were also recorded: (a) an overall echo score $(1-5+)$ given blindly by two echocardiographers, (b) the maximal proximal jet width by colour Doppler, (c) left ventricular end systolic and end diastolic volumes and left ventricular mass. The regurgitant area ranged from 0.25 to $1.7 \mathrm{~cm}^{2}$ and this area accorded with the overall echo score and the maximal proximal jet width measured by colour Doppler.

The aortic regurgitation orifice area can be calculated non-invasively and it may be a quantitative measure of the severity of aortic regurgitation.
\end{abstract}

(Br Heart J 1992;68:236-40)

Doppler echocardiography is both sensitive and specific for the detection of aortic regurgitation, ${ }^{12}$ but quantification of the severity of aortic regurgitation has proved difficult. Earlier studies showed correlation between the severity of aortic regurgitation and various non-invasive assessments by pulsed wave Doppler flow mapping, ${ }^{1-3}$ regurgitant fractions, ${ }^{45}$ pressure half-time, ${ }^{67}$ and colour flow mapping, ${ }^{8}$ but none of these methods is load independent and each has its limitations. Even the current reference standard of aortography is dependent on heart rate, blood pressure, and ventricular compliance.

As well as loading conditions, the clinical and haemodynamic consequences of aortic regurgitation also depend upon the size of the regurgitant orifice and this area is theoretically independent of the haemodynamic conditions under which it is measured and thus may be useful clinically. When the valve orifice area in aortic stenosis was calculated non-invasively by the continuity principle it correlated closely with the valve area calculated at cardiac catheterisation by the Gorlin formula. ${ }^{9-11}$ In this study, we investigate the feasibility of using the continuity equation in diastole to calculate the aortic regurgitant orifice area non-invasively in patients with aortic regurgitation and to examine the relation between this regurgitant area and the currently used non-invasive measures of the severity of aortic regurgitation.

\section{Patients and methods}

STUDY POPULATION

We studied 20 patients with a clinical diagnosis of aortic regurgitation referred for diagnostic echocardiography at Brigham and Women's Hospital in whom the clinical diagnosis of aortic regurgitation was substantiated by cross sectional Doppler echocardiography. Patients with associated aortic stenosis with a peak instantaneous systolic gradient greater than $30 \mathrm{~mm} \mathrm{Hg}$ were excluded. Patients with atrial fibrillation were also excluded.

\section{DATA ACQUISITION}

Cross sectional Doppler echocardiograms were recorded with simultaneous electrocardiography on a Hewlett Packard ultrasonoscope with a $2.5 \mathrm{MHz}$ imaging transducer and a 1.9 $\mathrm{MHz}$ Doppler transducer.

Cross sectional echocardiographic images were obtained of $(a)$ the left ventricular short axis view at the level of the high papillary muscles from the left parasternal region and the apical four chamber view to calculate left ventricular volumes and left ventricular mass and of $(b)$ the left ventricular outflow tract and aorta from the left parasternal region to 
measure the diameters of the aorta at the level of the annulus and $2 \mathrm{~cm}$ above the valve.

Pulsed wave Doppler velocity signals were recorded from the proximal aorta $2 \mathrm{~cm}$ above the aortic valve leaflets during diastole from either the apical long axis or the apical five chamber view. The Doppler signals were corrected for the angle of the aorta. Continuous wave Doppler velocity signals of the regurgitant jet were recorded from the left ventricular apex or the second right intercostal space. The highest velocity signal from one of the views was recorded. Doppler colour flow mapping was obtained of $(a)$ the left parasternal long axis view of the left ventricle to assess the width of the regurgitant aortic jet at the level of the aortic leaflets and (b) the apical left ventricular long axis and the apical five chamber views to assess the length of the regurgitant jet from the aortic valve and the width of the jet at valve level.

\section{DATA ANALYSIS}

Doppler velocity signals and two dimensional images were transferred from standard $1.2 \mathrm{~mm}$ videotape to the hard disc of an offline computer analysis system (GTI Freeland Medical Division, Indianapolis, Minnesota). For each patient, three consecutive pulsed wave Doppler velocity signals recorded from the supravalvar level ( $2 \mathrm{~cm}$ above the aortic leaflets) in diastole and three continuous wave Doppler velocity signals of the regurgitant jet were digitised to obtain the velocity time integrals, $\mathrm{VTI}_{s}$ and $\mathrm{VTI}_{\mathfrak{j}}$ respectively, and the mean values for each were calculated.

The cross sectional echocardiographic images of the long axis of the aorta and left ventricular outflow tract from the left parasternal region were digitised. We used a cross-hair electronic cursor to measure the supra-aortic diameter during three consecutive cardiac cycles at the beginning of diastole (that is, concordant with the $T$ wave on the electrocardiogram) $2 \mathrm{~cm}$ above the annulus - the point at which the supra-aortic pulsed wave Doppler velocity signals were recorded. We used the leading edge to leading edge convention for these measurements. ${ }^{12}$ Mean values for aortic diameter were obtained and used to calculate the supravalvar cross sectional area $\left(A_{s}\right)$, assuming a circular cross section. Blood volume flows were calculated as the product of the velocity-time integrals (VTI) and cross sectional areas (A) of the flow streams. The continuity equation states that flow at the regurgitant orifice equals flow at supravalvar level thus:

Aortic regurgitant orifice area $\left(\mathbf{A}_{\mathbf{j}}\right) \times$ velocity-time integral $\left(\mathrm{VTI}_{\mathrm{j}}\right)=$ supravalvar cross sectional area $\left(A_{s}\right) \times$ velocity-time integral $\left(\mathrm{VTI}_{\mathrm{s}}\right)$

Thus the jet orifice area can be obtained as:

$$
A_{j}=\frac{A_{s} \times V_{T I}}{V^{s} I_{j}}
$$

Two experienced clinical echocardiographers who had no knowledge of the calculated regurgitant areas made an overall Doppler echocardiographic assessment of the aortic regurgitation. This assessment combined the size of the left ventricle, the lengths of the colour flow jets, continuous wave (pressure half time), and pulsed wave Doppler mapping with an overall arbitrary scale from 1 to $5+$ (grade 5 was the most severe). The width of the aortic regurgitant jet was also estimated by colour flow mapping at the level of the aortic valve leaflets in the parasternal long axis, the apical long axis, and in the apical five chamber views. The maximal jet widths were measured.

Cross sectional echocardiographic images of the left ventricular short axis at high papillary muscle level and apical four chamber views were digitised. End diastolic and end systolic ventricular volumes, ejection fraction, and left ventricular mass were calculated as previously described. ${ }^{1314}$

\section{STATISTICAL ANALYSIS}

The values of the non-invasive measurements in individual patients were calculated as the means of three consecutive cardiac cycles. Results were reported for the group as mean (SE) and range. The method of Bland and Altman $^{15}$ was used to assess the agreement between the regurgitant area method and various non-invasive methods for assessing the severity of aortic regurgitation. In order to do the appropriate calculation, the regurgitant area was also categorised as 1 to $5+$ in order to compare with similar numeric grading scale ( 1 to $5+$ ) in the overall echo score. Univariate analysis by linear regression was used to compare the area with the ventricular volumes. Patients with significant mitral regurgitation $(>1+)$ were excluded from volume and mass correlations.

\section{Results}

PATIENTS' CHARACTERISTICS

The mean age of the patients was 55 years (range 22-82 years). There were 12 male and eight female patients. The aetiology of the aortic regurgitation was aortic dissection in one, systemic lupus erythematosus in two, rheumatic disease in six, and unknown in 11 . Four patients had mild aortic stenosis (gradients $<30 \mathrm{~mm} \mathrm{Hg}$ ), six had mild to moderate mitral regurgitation, and one had mild mitral stenosis and regurgitation. Twelve of the 20 patients were in New York Heart Association class I, six were in class II, and two were in class III failure. Eight out of the 20 patients were on afterload reducing agents when the echocardiography was performed.

\section{ECHOCARDIOGRAPHY}

Figure 1 shows representative Doppler signals from one patient. The supravalvar velocitytime integral (fig 1A) obtained in the apical long axis view showed aliasing of the forward blood flow during systole, while regurgitant flow towards the transducer had a peak velocity of $60 \mathrm{~cm} / \mathrm{s}$. The velocity declined through diastole as the pressures in the aorta and left ventricle approached each other. The velocity-time integral (VTI) of the regurgitant orifice in the 
Figure 1 Pulsed and continuous Doppler measurements for calculating the aortic regurgitant orifice of one patient. (A) Pulsed Doppler signal at the supravalvar level obtained from the apical long axis view. (B) Continuous wave Doppler signal at the regurgitant orifice level obtained from the apex. (C) Forward and regurgitant Doppler signals obtained in the aortic arch. The Doppler spectral recordings are plotted with time on the $x$ axis ( 1 s time lines) velocity on the $y$ axis (calibrations on the right are in $\mathrm{cm} / \mathrm{s}$ )
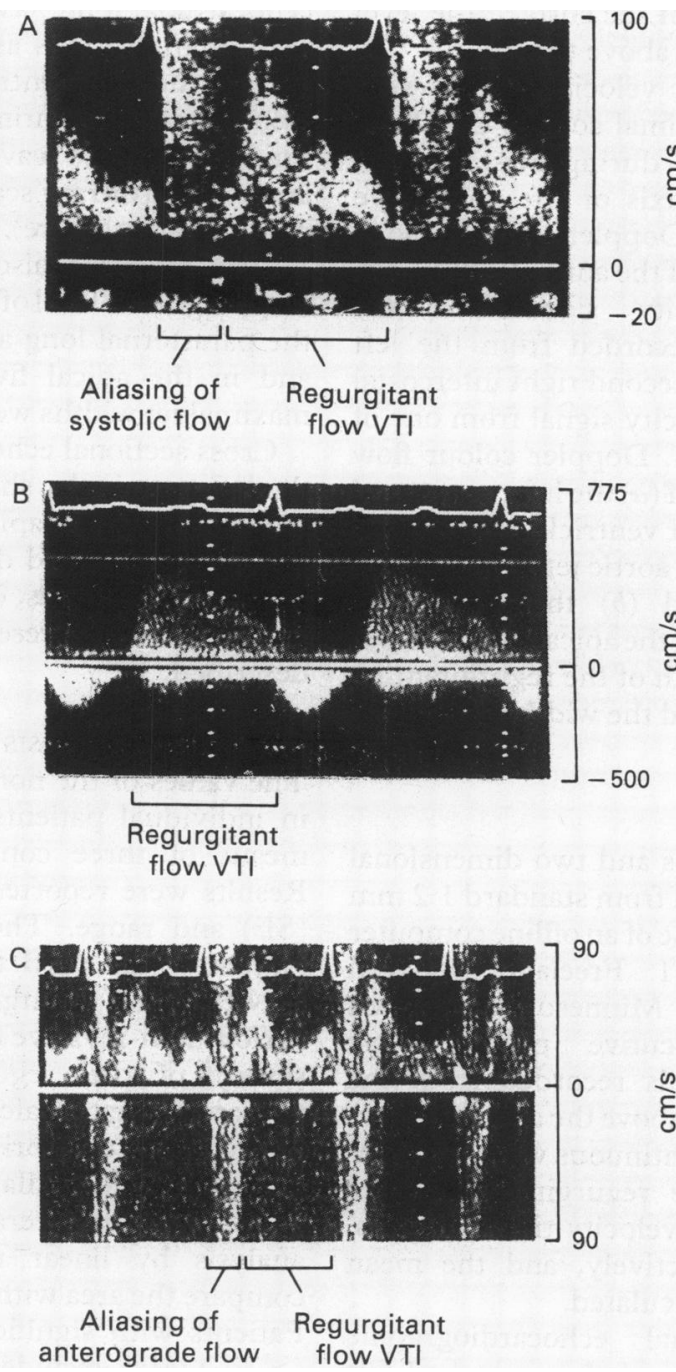

same patient was obtained with continuous wave Doppler as shown in fig 1B. The VTI had a well defined border although its decay did not seem to be linear. The peak velocity was approximately $3.5 \mathrm{~m} / \mathrm{s}$, and at the end of diastole the velocity was approximately $2.0 \mathrm{~m} / \mathrm{s}$. Figure $1 \mathrm{C}$ shows the forward and regurgitant flow Doppler signals obtained in the aortic arch by pulsed Doppler. The forward component had a relatively high velocity with aliasing, while the regurgitant component had a peak velocity of $70-80 \mathrm{~cm} / \mathrm{s}$.

In these twenty patients, the mean aortic regurgitant orifice area calculated by the continuity equation was $0.65 \mathrm{~cm}^{2}$ (range 0.25 $1.7 \mathrm{~cm}^{2}$ ). Overall, the regurgitant orifice area represented from 4 to $26 \%$ of the aortic annulus cross sectional area. Table 1 lists the results of various non-invasive measurements. Four patients with mitral regurgitation of
Table 1 Mean and range of the aortic regurgitation orifice area and the ventricular volumes/mass

Variable

AR orifice area $\left(\mathrm{cm}^{2}\right)$ :

Mean $0.65(0.08)$

$\begin{array}{ll}\text { Mean } & 0.65(0.08) \\ \text { Range } & 0.25 \text { to } 1.73\end{array}$

AR orifice area/annulus area (\%):

Mean

Range

Maximal proximal jet width $(\mathrm{cm})$ :

Mean

$12 \cdot 7$

Range

4 to 26

End diastolic volume (ml):

Mean

$1 \cdot 1(0 \cdot 1)$

0.6 to 1.8

Range

$277(33)$

End systolic volume $(\mathrm{ml})$ :

Mean

$100(734)$

$46(424)$

Left ventricular mass $(g)$ :

Mean

Range

$351(23)$

AR, aortic regurgitation

$>1+$ were excluded from volume and mass calculations.

Table 2 shows the results of Bland and Altman analysis. The mean differences between the regurgitant area and the overall echo score was 0.4 with $95 \%$ confidence interval of -0.2 to $1 \cdot 0$, though the limits of agreement are large, -2 to +3 grade. The diameter derived from the regurgitant area (assuming a circular orifice, $2 \sqrt{\operatorname{area} / \pi}$ ) agreed well with the maximal jet width with small limits of agreement. However, there was a systematic "overestimation" of $1.0 \mathrm{~cm}$ by the colour jet mapping. Table 2 shows that there were wide limits of agreement even between the various non-invasive indices in the same patients.

The end systolic and end diastolic volumes (16 patients) correlated significantly with the regurgitant orifice area, with $p$ values of 0.01 and 0.006 respectively (fig 2 ). The left ventricular mass did not correlate with the regurgitant orifice area.

\section{Discussion}

Non-invasive evaluation of aortic regurgitation has been largely qualitative, which contrasts with the quantitative measurement obtained in aortic stenosis by measuring the pressure gradient and the valve area derived from the continuity equation. The method we describe used the same principle as the calculation of the valve area in aortic stenosis, except that the Doppler velocity-time integrals were measured during diastole.

The regurgitant area measured by the continuity equation method is consistent with the clinical and historical impression that mild

Table 2 Statistical significance of non-invasive indices of aortic regurgitation by the method of Bland and Altman

\begin{tabular}{|c|c|c|c|c|}
\hline & $\begin{array}{l}\text { Differences } \\
\text { (D) }\end{array}$ & $\begin{array}{l}\text { Standard } \\
\text { error } \\
(S E)\end{array}$ & $\begin{array}{l}d \\
\quad 95 \% C I \text { of } D\end{array}$ & $\begin{array}{l}\text { Limits of } \\
\text { agreement }\end{array}$ \\
\hline $\begin{array}{l}\text { Regurgitant area }(1 \text { to } 5+) v \text { overall echo score }(1 \text { to } 5+) \\
\text { Regurgitant diameter }(\mathrm{cm}) v \text { colour jet width }(\mathrm{cm}) \\
\text { Overall echo score }(1 \text { to } 5+) v \text { colour jet length }(1 \text { to } 5+) \\
\text { Overall echo score }(1 \text { to } 5+) v \text { pressure } 1 / 2 \text { time }(1 \text { to } 5+)\end{array}$ & $\begin{array}{r}0.4 \\
1.0 \\
-0.6 \\
-0.4\end{array}$ & $\begin{array}{l}0.3 \\
0 \cdot 1 \\
0 \cdot 2 \\
0.5\end{array}$ & $\begin{array}{r}-0.2 \text { to } 1.0 \\
0.9 \text { to } 1 \cdot 1 \\
-1.0 \text { to } 0.2 \\
-1.3 \text { to } 0.6\end{array}$ & $\begin{array}{l}-2 \text { to } 3 \\
0.5 \text { to } 1.5 \\
-2.4 \text { to } 1 \cdot 2 \\
-4.5 \text { to } 3.8\end{array}$ \\
\hline
\end{tabular}

CI, confidence intervals. 
Figure 2 Relation between the aortic regurgitant orifice area and the end systolic (ESV) and end diastolic volumes (EDV) $(n=16)$ (patients with mitral regurgitation excluded) Univariate analysis with linear regression showed a significant correlation between the area and the two volumes ( $E S V$ : $r=0.61, p<0.01$; $E D V: r=0.65$ $p<0.006$ ).

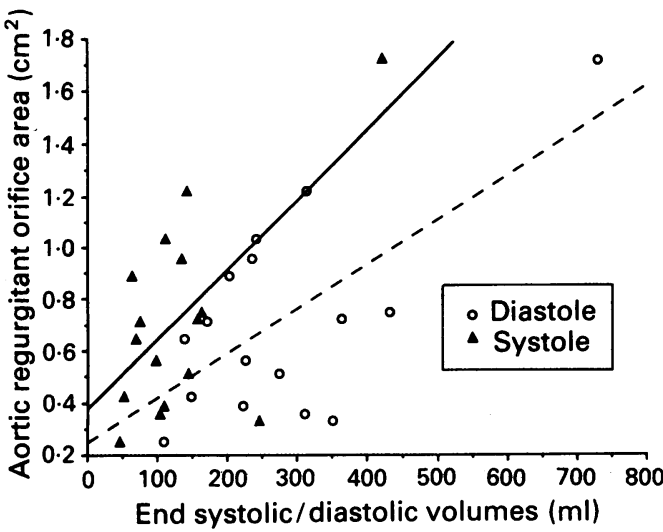

aortic regurgitation is associated with an orifice that occupies $5-10 \%$ of the aortic valve, that is 0.2 to $0.5 \mathrm{~cm}^{2} .{ }^{16}$ Severe aortic regurgitation usually results from a regurgitation orifice of more than 20 to $25 \%$ of the valve area (that is $>1.0 \mathrm{~cm}^{2}$ ). These data are consistent with our calculated regurgitant area of 0.25 to $1.7 \mathrm{~cm}^{2}$.

Because only four of our study patients required diagnostic cardiac catheterisation, the generally accepted reference standard of cineaortography grading was not available for most patients. However, it is clear that cineaortography is at best semiquantitative, and greatly depends on heart rate, afterload, and ventricular size. As a substitute, we used an overall echo score for comparison. This score is a composite non-invasive measure of the severity of the aortic regurgitation, as assessed by two experienced echocardiographers who were unaware of the calculated regurgitant orifice areas. The regurgitant areas calculated by the continuity equation accorded well with this score, suggesting that this orifice area relates to the overall severity of the aortic insufficiency. The agreement between this regurgitant area and the overall score is no worse than that for other more established indices of aortic regurgitation (that is, the pressure $1 / 2$ time and colour jet length).

The maximal proximal jet width as determined by colour Doppler accorded with the regurgitant area derived diameter obtained by the continuity equation. However, there was consistent overestimation by colour jet mapping. This difference was probably the result of (a) the spray effect of the jet, ${ }^{17}(b)$ the nonperpendicular angle at which the colour Doppler beam bisects the jet, and (c) the fact that the area determined by the continuity equation corresponds to the area of the vena contracta and is smaller than the true anatomical regurgitant area.

When patients with clinically important mitral regurgitation were excluded, left ventricular end diastolic and end systolic volumes correlated with aortic regurgitation orifice area. However, there was a degree of variability in these relations because left ventricular volumes depend on both the severity and the chronicity of the regurgitation, and the same holds true for the left ventricular mass.

The advantage of calculating the regurgitant orifice area to determine the severity of aortic regurgitation is that it is theoretically independent of changes in heart rate, pressure gradient, or left ventricular compliance. However, these assumptions will need to be confirmed clinically in a larger study. Regurgitant orifice areas may be useful in several settings: $(a)$ in mixed aortic valve disease when the regurgitant orifice area will help to define the aortic regurgitation component of the transvalvar gradient-(thus correcting the underestimation of the stenotic aortic valve area that commonly occurs in noninvasive evaluation of mixed aortic valvar disease), $(b)$ in following the patients with chronic aortic regurgitation, in whom the change in orifice area may indicate an insidious increase in severity before the onset of symptoms or a change in left ventricular size and function; (c) in aortic regurgitation, when knowing the regurgitant orifice area may provide insight into the mechanism and degree of aortic regurgitation which predictably results in progressive left ventrticular dilatation and decompensation.

\section{LIMITATIONS OF THE STUDY}

The assumption that coronary flow makes a negligible contribution to the regurgitation velocity-time integral may artificially slightly increase the regurgitant orifice area. This error is estimated to be of the order of less than $10 \%$. The continuity equation assumes that the supravalvar retrograde flow has a flat velocity profile. It has been shown in an in vitro model that this diastolic velocity profile $2 \mathrm{~cm}$ above the valve has a blunted profile as long as the ascending aorta is less than $4.8 \mathrm{~cm}$ in diameter. ${ }^{18}$ Thus the Doppler signal obtained above the valve should represent the mean diastolic velocity in the aorta and not the increased velocities in the zone of flow convergence. The supravalvar velocity-time integral may be technically difficult to obtain in some patients because of their body habitus. Operators need practice to obtain adequate supravalvar signals.

We demonstrated the feasibility of calculating the regurgitant orifice area non-invasively using the continuity equation and we found that this area agrees with the current noninvasive techniques for assessing the severity of the aortic regurgitation. Further studies are required to define its reproducibility and its relation to the invasive assessment of aortic regurgitation.

1 Quinones MA, Young JB, Waggoner AD, Ostojic MC, Ribeiro LGT, Miller RR. Assessment of pulsed Dopple echocardiography in detection and quantification of aortic and mitral regurgitation. Br Heart J 1980;44:612-20.

2 Ciobanu M, Abbasi A, Allen M, Hermer A, Spellberg $R$ Pulsed Doppler echocardiograph in the diagnosis and estimation of severity of aortic insufficiency. $A m J$ Cardio 1982;49:339-43.

3 Masuyama T, Kitabatake A, Kodama $K$, Uematsu $M$ Nakatani S, Kamada T. Semiquantitative evaluation of aortic regurgitation by Doppler echocardiography: effects of associated mitral stenosis. Am Heart J 1989;117:133-9.

4 Kitabatake $A$, Ito $H$, Inoue $M$, et al. A new approach to noninvasive evaluation of aortic regurgitant fraction by two-dimensional Doppler echocardiography. Circulation 1985;72:523-9.

5 Touche T, Prasquier R, Nitenberg A de ZD, Gourgon R. Assessment and follow-up of patients with aortic regurgitation by an updated Doppler echocardiographic 
measurement of the regurgitant fraction in the aortic arch. Circulation 1985;72:819-24.

6 Graybum $P$, Handshow R, Smith M, Harrison M, DeMaria A. Quantitative assessment of the hemodynamic consequences of aortic regurgitation by means of continuous wave Doppler recordings. J Am Coll Cardiol 1987;10: 135-41.

7 Masuyama T, Kodama K, Kitabatake A, et al. Noninvasive evaluation of aortic regurgitation by continuous-wave Doppler echocardiography. Circulation 1986;73:460-6.

8 DallAglio V, DAngelo G, Moro E, et al. Interobservor and echo-angio variability of two-dimensional colour Dopple evaluation of aortic and mitral regurgitation. Eur Heart $J$ 1989;10:334-40.

9 Richards K, Cannon S, Miller J, Crawford M. Calculation of aortic valve area by Doppler echocardiography: a direct application of the continuity equation. Circulation 1986;73:964-9.

10 Otto CM, Pearlman AS, Comess KA, Reamer RP, Janko CL, Huntsman IL. Determination of the stenotic aortic valve area in adults using Doppler echocardiography. valve area in adults using Dop
$J$ Am Coll Cardiol 1986;7:509-17.

11 Grayburn PA, Smith MD, Harrison MR, Gurley JC, DeMaria AN. Pivotal role of aortic valve area calculatio by the continuity equation for Doppler assessment of aortic stenosis in patients with combined aortic stenosis and regurgitation. Am J Cardiol 1988;61:376-81.
12 Sahn DJ, DeMaria A, Kisslo J, Weyman A. Recommendations regarding quantitation in M-mode echocardio-
graphy: Results of a survey of echocardiographic graphy: Results of a survey of echocarc

13 St John Sutton MG, Plappert TA, Hirshfeld JW, Reichek $\mathrm{N}$. Assessment of left ventricular mechanics in patients with asymptomatic aortic regurgitation: a two-dimensional echocardiographic study. Circulation 1984;69: 259-68.

14 Reichek N, Helak J, Plappert T, Sutton MS, Weber KT Anatomic validation of left ventricular mass estimates from clinical two-dimensional echocardiography: initial results. Circulation 1983;67:348-52.

15 Bland J, Altman D. Statistical methods for assessing agreement between two methods of clinical measurement. Lancet 1986; i:307-10.

16 Grossman W. Cardiac Catheterization and angiography. 3rd

ed. Philadelphia: Lea \& Febiger, 1986:374.
17 Switzer D, Yoganathan A, Nanda N, Woo Y, Ridgeway A. Calibration of colour Doppler flow mapping during
Cow Calibration of colour Doppler flow mapping during for a reliable quantitative grading system for aortic for a reliable quantitative grading system

18 Reimold SC, Ganz P, Bittl J, et al. Effective aortic regurgitant orifice area: description of a method based on the conservation of mass. J Am Coll Cardiol 1991;18:761-9. 